\title{
A novel exact solution for the fractional Ambartsumian equation
}

\author{
Abdelhalim Ebaid ${ }^{1 *}$, Carlo Cattani ${ }^{2}$, Amnah S. Al Juhani ${ }^{1}$ and Essam R. El-Zahar ${ }^{3,4}$
}

\section{"Correspondence:}

aebaid@ut.edu.sa;

halimgamil@yahoo.com

'Department of Mathematics,

Faculty of Science, University of

Tabuk, P.O. Box 741, Tabuk, 71491

Saudi Arabia

Full list of author information is

available at the end of the article

\begin{abstract}
Fractional calculus (FC) is useful in studying physical phenomena with memory effect. In this paper, a fractional form of Ambartsumian equation is considered utilizing the Caputo fractional derivative. The Heaviside expansion formula in classical calculus (CC) is extended/developed in view of FC. Then, the extended Heaviside expansion formula is applied to obtain the exact solution in a simplest form. Several theorems and lemmas are proved to facilitate the evaluation of the inverse Laplace transform of specific expressions in fractional forms. The exact solution is established in terms of a one-parameter Mittag-Leffler function which is provided for the first time for the Ambartsumian equation in FC. The present solution reduces to the corresponding one in the relevant literature as the fractional order tends to one. Moreover, the convergence of the obtained solution is theoretically proved. Comparisons with another approach in the literature are performed. The advantage of the present analysis over the existing one in the relevant literature is discussed and analyzed.
\end{abstract}

Keywords: Ambartsumian equation; Adomian decomposition method; Laplace transform; Fractional calculus; Mittag-Leffler function; Exact solution

\section{Introduction}

The standard Ambartsumian equation (SAE) was derived by Ambartsumian [1] more than two decades ago. This equation describes the absorption of light by the interstellar matter. In this paper, we consider the fractional Ambartsumian equation (FAE) in the form:

$$
{ }_{0}^{C} D_{t}^{\alpha} y(t)=-y(t)+\frac{1}{\xi} y\left(\frac{t}{\xi}\right), \quad 0<\alpha \leq 1, \xi>1,
$$

where $\xi$ is a constant and $\alpha$ is the arbitrary order of the Caputo fractional derivative with the following initial condition (IC):

$$
y(0)=\lambda
$$

The FAE reduces to the SAE as $\alpha \rightarrow 1$. The SAE has been investigated by Kato and McLeod [2] for existence and uniqueness. Later, Patade and Bhalekar [3] solved the SAE using the power series approach, and the obtained power series solution was proved for conver-

(c) The Author(s) 2021. This article is licensed under a Creative Commons Attribution 4.0 International License, which permits use, sharing, adaptation, distribution and reproduction in any medium or format, as long as you give appropriate credit to the original author(s) and the source, provide a link to the Creative Commons licence, and indicate if changes were made. The images or other third party material in this article are included in the article's Creative Commons licence, unless indicated otherwise in a credit line to the material. If material is not included in the article's Creative Commons licence and your intended use is not permitted by statutory regulation or exceeds the permitted use, you will need to obtain permission directly from the copyright holder. To view a copy of this licence, visit http://creativecommons.org/licenses/by/4.0/. 
gence. In addition, Bakodah and Ebaid [4] obtained the exact solution for the SAE. Recently, Alatawi et al. [5] applied the homotopy perturbation method (HPM) to obtain the approximate solution of the SAE in terms of the exponential functions, while Khaled et al. [6] provided the solution using the conformable derivative. Very recently, Kumar et al. [7] obtained the approximate solution for the FAE using the homotopy transform analysis method (HTAM). It can be observed from Ref. [7] that the series solution is expressed in terms of $t^{\alpha}$ which converges in certain subdomains.

The objective of this paper is to obtain the exact solution of the FAE in terms of the oneparameter Mittag-Leffler function which converges in the whole domain $t \in[0, \infty)$. Our approach utilizes the Laplace transform (LT) combined with the Adomian decomposition method (ADM) [8-12]. The ADM [8-12] has been extensively used to solve various integral/differential equations and IVPs/BVPs [13-27]. The FC approach has been extended successfully to include several phenomena in physics, engineering, and biology [28-38]. In order to achieve the target of this paper, the Heaviside expansion formula in CC is extended in view of FC. The extended Heaviside expansion formula is applied to calculate the inverse LT of specific fractional expressions. Furthermore, it is shown that the present exact solution reduces to the corresponding one in the relevant literature as $\alpha \rightarrow 1$. Besides, the convergence of the present solution is theoretically proved. Moreover, numerical comparisons with the existing approach in the literature are performed to indicate the advantage and effectiveness of the present analysis.

\section{Preliminaries and analysis}

The Riemann-Liouville fractional integral of order $\alpha$ is defined as follows [39]:

$$
{ }_{0} I_{t}^{\alpha} y(t)=\frac{1}{\Gamma(\alpha)} \int_{0}^{t} \frac{y(\tau)}{(t-\tau)^{1-\alpha}} d \tau, \quad \alpha>0, t>0
$$

Let $\alpha \neq 0$ denote the order of the derivative in such a way that $n-1<\alpha \leq n$. Then the Caputo fractional derivative of a function $y(t)$ is defined by [39]

$$
{ }_{0}^{C} D_{t}^{\alpha} y(t)=\frac{d^{\alpha} y(t)}{d t^{\alpha}}= \begin{cases}\frac{1}{\Gamma(n-\alpha)} \int_{0}^{t}(t-\tau)^{n-\alpha-1} y^{(n)}(\tau) d \tau & \text { if } n-1<\alpha<n, \\ \frac{d^{n} y(t)}{d t^{n}} & \text { if } \alpha=n .\end{cases}
$$

In applied problems, it is required to use the definitions of fractional derivatives that allow the utilization of interpreted initial conditions. It is clear from Eq. (5) that definition (4) satisfies these demands

$$
L\left\{\frac{d^{\alpha} y(t)}{d t^{\alpha}}\right\}=s^{\alpha} Y(s)-\sum_{m=0}^{n-1} s^{\alpha-m-1} y^{(m)}(0)
$$

where $Y(s)$ is the Laplace transform (LT) of $y(t)$. When solving fractional differential equations, the following relations for the inverse LT in terms of Mittag-Leffler functions can be used, see [39] for details:

$$
\begin{aligned}
& L^{-1}\left\{\frac{m ! s^{\alpha-\beta}}{\left(s^{\alpha} \mp a\right)^{m+1}}\right\}=t^{\alpha m+\beta-1} E_{\alpha, \beta}^{(m)}\left( \pm a t^{\alpha}\right), \\
& L^{-1}\left\{\frac{s^{\alpha-1}}{s^{\alpha}+1}\right\}=E_{\alpha}\left(-t^{\alpha}\right),
\end{aligned}
$$




$$
\begin{aligned}
& L^{-1}\left\{\frac{1}{s^{\alpha}+a}\right\}=t^{\alpha-1} E_{\alpha, \alpha}\left(-a t^{\alpha}\right), \\
& L^{-1}\left\{\frac{s^{-1}}{s^{\alpha}+a}\right\}=t^{\alpha} E_{\alpha, \alpha+1}\left(-a t^{\alpha}\right),
\end{aligned}
$$

where the Mittag-Leffler functions of one parameter and two parameters are defined by

$$
\begin{aligned}
& E_{\alpha}(z)=\sum_{m=0}^{\infty} \frac{z^{m}}{\Gamma(\alpha m+1)}, \quad \alpha>0 \\
& E_{\alpha, \beta}(z)=\sum_{m=0}^{\infty} \frac{z^{m}}{\Gamma(\alpha m+\beta)} \quad(\alpha>0, \beta>0) .
\end{aligned}
$$

Some useful properties are given by

$$
\begin{aligned}
& E_{\alpha, \beta}(z)=z E_{\alpha, \alpha+\beta}(z)+\frac{1}{\Gamma(\beta)}, \\
& E_{\alpha}(z)=z E_{\alpha, \alpha+1}(z)+1, \\
& E_{\alpha, 1}(z)=E_{\alpha}(z), \quad E_{1}(z)=e^{z},
\end{aligned}
$$

and

$$
\begin{gathered}
\int_{0}^{t} \tau^{\gamma-1} E_{\alpha, \gamma}\left(a \tau^{\alpha}\right)(t-\tau)^{\beta-1} E_{\alpha, \beta}\left[b(t-\tau)^{\alpha}\right] d \tau \\
=\frac{t^{\beta+\gamma-1}}{a-b}\left[a E_{\alpha, \beta+\gamma}\left(a t^{\alpha}\right)-b E_{\alpha, \beta+\gamma}\left(b t^{\alpha}\right)\right] .
\end{gathered}
$$

The Heaviside expansion formula in CC is a well-known formula which is frequently used to calculate the inverse LT of specific expressions, the statement of such a formula is introduced below.

Theorem 1 (Heaviside expansion formula in CC) Let $H(s)$ and $G(s)$ be two polynomials such that the degree of $H(s)$ is less than the degree of $G(s)$, also assume that $G(s)$ has $n$ distinct zeros $\sigma_{k}, k=1,2,3, \ldots, n$, then

$$
L^{-1}\left\{\frac{H(s)}{G(s)}\right\}=\sum_{k=1}^{n} \frac{H\left(\sigma_{k}\right)}{G^{\prime}\left(\sigma_{k}\right)} \times e^{\sigma_{k} t}
$$

Proof See please Ref. [40] (pages 61-62).

\section{Analysis}

In this section, the Heaviside expansion formula is extended, and a generalized form of Eq. (16) is derived by the next theorem.

Theorem 2 (Extended Heaviside expansion formula) Let $0<\alpha \leq 1$ and suppose that $H\left(s^{\alpha}\right)$ and $G\left(s^{\alpha}\right)$ are two polynomials in $s^{\alpha}$ such that the degree of $H\left(s^{\alpha}\right)$ is less than the degree of $G\left(s^{\alpha}\right)$. If $G\left(s^{\alpha}\right)$ has $n$ distinct zeros $\sigma_{k}, k=1,2,3, \ldots, n$, then

$$
L^{-1}\left\{\frac{H\left(s^{\alpha}\right)}{G\left(s^{\alpha}\right)}\right\}=t^{\alpha-1} \sum_{k=1}^{n} \frac{H\left(\sigma_{k}\right)}{G^{\prime}\left(\sigma_{k}\right)} \times E_{\alpha, \alpha}\left(\sigma_{k} t^{\alpha}\right) .
$$


Proof Since $G\left(s^{\alpha}\right)$ is a polynomial with $n$ distinct zeros $\sigma_{1}, \sigma_{2}, \ldots \ldots, \sigma_{n}$, then we can write $\frac{H\left(s^{\alpha}\right)}{G\left(s^{\alpha}\right)}$ according to the method of partial fractions as follows:

$$
\frac{H\left(s^{\alpha}\right)}{G\left(s^{\alpha}\right)}=\frac{c_{1}}{s^{\alpha}-\sigma_{1}}+\frac{c_{2}}{s^{\alpha}-\sigma_{2}}+\cdots+\frac{c_{k}}{s^{\alpha}-\sigma_{k}}+\cdots+\frac{c_{n}}{s^{\alpha}-\sigma_{n}} .
$$

Multiplying both sides of Eq. (18) by $s^{\alpha}-\sigma_{1}$ and letting $s^{\alpha} \rightarrow \sigma_{1}$, we find, using L'Hospital's rule,

$$
\begin{aligned}
c_{1} & =\lim _{s^{\alpha} \rightarrow \sigma_{1}}\left(s^{\alpha}-\sigma_{1}\right) \frac{H\left(s^{\alpha}\right)}{G\left(s^{\alpha}\right)}, \\
& =\lim _{s^{\alpha} \rightarrow \sigma_{1}} H\left(s^{\alpha}\right) \times \lim _{s^{\alpha} \rightarrow \sigma_{1}}\left\{\frac{s^{\alpha}-\sigma_{1}}{G\left(s^{\alpha}\right)}\right\}, \\
& =H\left(\sigma_{1}\right) \times \lim _{s^{\alpha} \rightarrow \sigma_{1}}\left\{\frac{\frac{d}{d s^{\alpha}}\left(s^{\alpha}-\sigma_{1}\right)}{\frac{d}{d s^{\alpha}} G\left(s^{\alpha}\right)}\right\}, \\
& =H\left(\sigma_{1}\right) \times \lim _{s^{\alpha} \rightarrow \sigma_{1}}\left\{\frac{1}{G^{\prime}\left(s^{\alpha}\right)}\right\}, \\
& =\frac{H\left(\sigma_{1}\right)}{G^{\prime}\left(\sigma_{1}\right)} .
\end{aligned}
$$

Similarly, the general term $c_{k}$ can be calculated as follows:

$$
\begin{aligned}
c_{k} & =\lim _{s^{\alpha} \rightarrow \sigma_{k}}\left(s^{\alpha}-\sigma_{k}\right) \frac{H\left(s^{\alpha}\right)}{G\left(s^{\alpha}\right)}, \\
& =\lim _{s^{\alpha} \rightarrow \sigma_{k}} H\left(s^{\alpha}\right) \times \lim _{s^{\alpha} \rightarrow \sigma_{k}}\left\{\frac{s^{\alpha}-\sigma_{k}}{G\left(s^{\alpha}\right)}\right\}, \\
& =H\left(\sigma_{k}\right) \times \lim _{s^{\alpha} \rightarrow \sigma_{k}}\left\{\frac{\frac{d}{d s^{\alpha}}\left(s^{\alpha}-\sigma_{k}\right)}{\frac{d}{d s^{\alpha}} G\left(s^{\alpha}\right)}\right\}, \\
& =H\left(\sigma_{k}\right) \times \lim _{s^{\alpha} \rightarrow \sigma_{k}}\left\{\frac{1}{G^{\prime}\left(s^{\alpha}\right)}\right\}, \\
& =\frac{H\left(\sigma_{k}\right)}{G^{\prime}\left(\sigma_{k}\right)} .
\end{aligned}
$$

Therefore, Eq. (18) can be expressed as

$$
\begin{aligned}
\frac{H\left(s^{\alpha}\right)}{G\left(s^{\alpha}\right)}= & \frac{H\left(\sigma_{1}\right)}{G^{\prime}\left(\sigma_{1}\right)} \frac{1}{s^{\alpha}-\sigma_{1}}+\frac{H\left(\sigma_{2}\right)}{G^{\prime}\left(\sigma_{2}\right)} \frac{1}{s^{\alpha}-\sigma_{2}}+\cdots+\frac{H\left(\sigma_{k}\right)}{G^{\prime}\left(\sigma_{k}\right)} \frac{1}{s^{\alpha}-\sigma_{k}}+\frac{H\left(\sigma_{n}\right)}{G^{\prime}\left(\sigma_{n}\right)}+\cdots \\
& +\frac{1}{s^{\alpha}-\sigma_{n}}
\end{aligned}
$$

or

$$
\frac{H\left(s^{\alpha}\right)}{G\left(s^{\alpha}\right)}=\sum_{k=1}^{n} \frac{H\left(\sigma_{k}\right)}{G^{\prime}\left(\sigma_{k}\right)} \times \frac{1}{s^{\alpha}-\sigma_{k}} .
$$


Applying the inverse LT on Eq. (22), we obtain

$$
L^{-1}\left\{\frac{H\left(s^{\alpha}\right)}{G\left(s^{\alpha}\right)}\right\}=\sum_{k=1}^{n} \frac{H\left(\sigma_{k}\right)}{G^{\prime}\left(\sigma_{k}\right)} \times L^{-1}\left\{\frac{1}{s^{\alpha}-\sigma_{k}}\right\},
$$

and this yields

$$
L^{-1}\left\{\frac{H\left(s^{\alpha}\right)}{G\left(s^{\alpha}\right)}\right\}=t^{\alpha-1} \sum_{k=1}^{n} \frac{H\left(\sigma_{k}\right)}{G^{\prime}\left(\sigma_{k}\right)} \times E_{\alpha, \alpha}\left(\sigma_{k} t^{\alpha}\right) .
$$

Lemma 1 The extended Heaviside expansion formula (17) reduces to the original Heaviside expansion formula (16) as $\alpha \rightarrow 1$.

Proof From Eq. (17) provided by Theorem 1, we have as $\alpha \rightarrow 1$ that

$$
L^{-1}\left\{\frac{H(s)}{G(s)}\right\}=\sum_{k=1}^{n} \frac{H\left(\sigma_{k}\right)}{G^{\prime}\left(\sigma_{k}\right)} \times E_{1,1}\left(\sigma_{k} t\right)=\sum_{k=1}^{n} \frac{H\left(\sigma_{k}\right)}{G^{\prime}\left(\sigma_{k}\right)} \times e^{\sigma_{k} t},
$$

which is the original Heaviside expansion formula (16).

Lemma 2 (Special case of the extended Heaviside expansion formula) If $H\left(s^{\alpha}\right)$ and $G_{i}\left(s^{\alpha}\right)$ are two polynomials in $s^{\alpha}$ such that

$$
H\left(s^{\alpha}\right)=s^{\alpha}, \quad G_{i}\left(s^{\alpha}\right)=\prod_{k=0}^{i}\left(s^{\alpha}+\xi^{-k \alpha}\right), \quad i \geq 1,0<\alpha \leq 1
$$

then

$$
L^{-1}\left\{\frac{H\left(s^{\alpha}\right)}{G_{i}\left(s^{\alpha}\right)}\right\}=t^{\alpha-1} \sum_{k=0}^{i} \frac{-\xi^{-k \alpha}}{G_{i}^{\prime}\left(-\xi^{-k \alpha}\right)} \times E_{\alpha, \alpha}\left(-\xi^{-k \alpha} t^{\alpha}\right) .
$$

Proof From the definitions of $H\left(s^{\alpha}\right)$ and $G_{i}\left(s^{\alpha}\right)$, it is clear that $H\left(s^{\alpha}\right)$ has a degree less than that of $G_{i}\left(s^{\alpha}\right) \forall i \geq 1$. Besides, $G_{i}\left(s^{\alpha}\right)$ has $i+1$ distinct zeros $\sigma_{k}=-\xi^{-k \alpha}, k=0,1,2,3, \ldots, i$. Applying Theorem 2 and substituting $\sigma_{k}=-\xi^{-k \alpha}$ into the extended Heaviside expansion formula yields

$$
L^{-1}\left\{\frac{H\left(s^{\alpha}\right)}{G_{i}\left(s^{\alpha}\right)}\right\}=t^{\alpha-1} \sum_{k=0}^{i} \frac{H\left(-\xi^{-k \alpha}\right)}{G_{i}^{\prime}\left(-\xi^{-k \alpha}\right)} \times E_{\alpha, \alpha}\left(-\xi^{-k \alpha} t^{\alpha}\right) .
$$

However, the definition of $H\left(s^{\alpha}\right)$ gives $H\left(-\xi^{-k \alpha}\right)=-\xi^{-k \alpha}$, hence,

$$
L^{-1}\left\{\frac{H\left(s^{\alpha}\right)}{G_{i}\left(s^{\alpha}\right)}\right\}=t^{\alpha-1} \sum_{k=0}^{i} \frac{-\xi^{-k \alpha}}{G_{i}^{\prime}\left(-\xi^{-k \alpha}\right)} \times E_{\alpha, \alpha}\left(-\xi^{-k \alpha} t^{\alpha}\right) .
$$

\section{The exact solution}

This section is devoted to obtaining the solution of FAE (1)-(2) in an exact form in terms of the Mittag-Leffler functions. The previous theorems and lemmas are applied in this section to derive such exact solution. 


\subsection{Solution in terms of two-parameter Mittag-Leffler function} Applying the LT on Eq. (1) and noting that $L\left\{\left(\frac{1}{\xi} y\left(\frac{t}{\xi}\right)\right)\right\}=Y(\xi s)$ yield

$$
Y(s)=\frac{\lambda s^{\alpha-1}}{s^{\alpha}+1}+\frac{Y(\xi s)}{s^{\alpha}+1} .
$$

The ADM assumes the solution of (30) in the series form

$$
Y(s)=\sum_{i=0}^{\infty} Y_{i}(s)
$$

which leads to

$$
\begin{aligned}
& Y_{0}(s)=\frac{\lambda s^{\alpha-1}}{s^{\alpha}+1}, \\
& Y_{i}(s)=\frac{Y_{i-1}(\xi s)}{s^{\alpha}+1}, \quad i \geq 1 .
\end{aligned}
$$

The recurrence scheme (33) gives

$$
\begin{aligned}
& Y_{1}(s)=\frac{Y_{0}(\xi s)}{s^{\alpha}+1}=\frac{\lambda(\xi s)^{\alpha-1}}{\left(s^{\alpha}+1\right)\left(\xi^{\alpha} s^{\alpha}+1\right)}=\frac{\lambda\left(\xi^{1} s\right)^{\alpha-1}}{\prod_{k=0}^{1}\left(\xi^{k \alpha} s^{\alpha}+1\right)}, \\
& Y_{2}(s)=\frac{Y_{1}(\xi s)}{s^{\alpha}+1}=\frac{\lambda\left(\xi^{2} s\right)^{\alpha-1}}{\left(s^{\alpha}+1\right)\left(\xi^{\alpha} S^{\alpha}+1\right)\left(\xi^{2 \alpha} s^{\alpha}+1\right)}=\frac{\lambda\left(\xi^{2} s\right)^{\alpha-1}}{\prod_{k=0}^{2}\left(\xi^{k \alpha} s^{\alpha}+1\right)}, \\
& Y_{3}(s)=\frac{Y_{2}(\xi s)}{s^{\alpha}+1}=\frac{\lambda\left(\xi^{3} s\right)^{\alpha-1}}{\left(s^{\alpha}+1\right)\left(\xi^{\alpha} S^{\alpha}+1\right)\left(\xi^{2 \alpha} s^{\alpha}+1\right)\left(\xi^{3 \alpha} s^{\alpha}+1\right)}=\frac{\lambda\left(\xi^{2} s\right)^{\alpha-1}}{\prod_{k=0}^{3}\left(\xi^{k \alpha} s^{\alpha}+1\right)},
\end{aligned}
$$

and hence the general component $Y_{i}(s)$ can be obtained as

$$
Y_{i}(s)=\frac{\lambda\left(\xi^{i} s\right)^{\alpha-1}}{\left(s^{\alpha}+1\right)\left(\xi^{\alpha} S^{\alpha}+1\right)\left(\xi^{2 \alpha} s^{\alpha}+1\right) \ldots \ldots\left(\xi^{i \alpha} S^{\alpha}+1\right)}, \quad i \geq 1 .
$$

Therefore, $Y_{i}(s)$ can be written as

$$
Y_{i}(s)=\frac{\lambda\left(\xi^{i} s\right)^{\alpha-1}}{\prod_{k=0}^{i}\left(\xi^{k \alpha} s^{\alpha}+1\right)}, \quad i \geq 1 .
$$

Also, we note that

$$
\prod_{k=0}^{i}\left(\xi^{k \alpha} S^{\alpha}+1\right)=\xi^{i(i+1) \alpha / 2} \prod_{k=0}^{i}\left(s^{\alpha}+\xi^{-k \alpha}\right) .
$$

Inserting (37) into (36) and simplifying lead to

$$
Y_{i}(s)=\lambda \xi^{i \alpha(1-i) / 2-i}\left(\frac{1}{s} \times \frac{s^{\alpha}}{\prod_{k=0}^{i}\left(s^{\alpha}+\xi^{-k \alpha}\right)}\right), \quad i \geq 1 .
$$

Assume that $H\left(s^{\alpha}\right)$ and $G_{i}\left(s^{\alpha}\right)$ are defined as in Lemma 2, then Eq. (38) is expressed as

$$
Y_{i}(s)=\lambda \xi^{i \alpha(1-i) / 2-i}\left(\frac{1}{s} \times \frac{H\left(s^{\alpha}\right)}{G_{i}\left(s^{\alpha}\right)}\right), \quad i \geq 1 .
$$


From (32), it then follows

$$
Y(s)=Y_{0}(s)+\sum_{i=1}^{\infty} Y_{i}(s)
$$

i.e.,

$$
Y(s)=\frac{\lambda s^{\alpha-1}}{s^{\alpha}+1}+\lambda \sum_{i=1}^{\infty} \xi^{i \alpha(1-i) / 2-i}\left(\frac{1}{s} \times \frac{H\left(s^{\alpha}\right)}{G_{i}\left(s^{\alpha}\right)}\right)
$$

Applying the inverse LT on the last equation, we get the solution $y(t)$ of the current model as

$$
y(t)=L^{-1}\left\{\frac{\lambda s^{\alpha-1}}{s^{\alpha}+1}\right\}+\lambda \sum_{i=1}^{\infty} \xi^{i \alpha(1-i) / 2-i}\left(L^{-1}\left\{\frac{1}{s}\right\} * L^{-1}\left\{\frac{H\left(s^{\alpha}\right)}{G_{i}\left(s^{\alpha}\right)}\right\}\right),
$$

where $(*)$ refers to the convolution operation. From the results of Lemma 2, we have

$$
y(t)=\lambda E_{\alpha}\left(-t^{\alpha}\right)+\lambda \sum_{i=1}^{\infty} \xi^{i \alpha(1-i) / 2-i}\left(1 *\left\{t^{\alpha-1} \sum_{k=0}^{i} \frac{-\xi^{-k \alpha}}{G_{i}^{\prime}\left(-\xi^{-k \alpha}\right)} \times E_{\alpha, \alpha}\left(-\xi^{-k \alpha} t^{\alpha}\right)\right\}\right)
$$

or

$$
y(t)=\lambda E_{\alpha}\left(-t^{\alpha}\right)+\lambda \sum_{i=1}^{\infty} \sum_{k=0}^{i} \xi^{i \alpha(1-i) / 2-i} \int_{0}^{t}\left(\tau^{\alpha-1} \frac{-\xi^{-k \alpha}}{G_{i}^{\prime}\left(-\xi^{-k \alpha}\right)} \times E_{\alpha, \alpha}\left(-\xi^{-k \alpha} \tau^{\alpha}\right)\right) d \tau,
$$

which can be written as

$$
y(t)=\lambda E_{\alpha}\left(-t^{\alpha}\right)+\lambda \sum_{i=1}^{\infty} \sum_{k=0}^{i} \xi^{i \alpha(1-i) / 2-i} \times \frac{-\xi^{-k \alpha}}{G_{i}^{\prime}\left(-\xi^{-k \alpha}\right)} \int_{0}^{t}\left(\tau^{\alpha-1} E_{\alpha, \alpha}\left(-\xi^{-k \alpha} \tau^{\alpha}\right)\right) d \tau .
$$

Using the integral formula (15) when $\gamma=\alpha, \beta=1, a=-\xi^{-k \alpha}$, and $b=0$, we obtain

$$
\int_{0}^{t} \tau^{\alpha-1} E_{\alpha, \alpha}\left(-\xi^{-k \alpha} \tau^{\alpha}\right) d \tau=t^{\alpha} E_{\alpha, \alpha+1}\left(-\xi^{-k \alpha} t^{\alpha}\right)
$$

From (45) and (46), we can write

$$
y(t)=\lambda E_{\alpha}\left(-t^{\alpha}\right)+\lambda \sum_{i=1}^{\infty} \sum_{k=0}^{i} \frac{\xi^{i \alpha(1-i) / 2-i}}{G_{i}^{\prime}\left(-\xi^{-k \alpha}\right)}\left(-\xi^{-k \alpha} t^{\alpha} E_{\alpha, \alpha+1}\left(-\xi^{-k \alpha} t^{\alpha}\right)\right) .
$$

\subsection{Solution in terms of one-parameter Mittag-Leffler function}

Implementing property (13) for $z=-\xi^{-k \alpha} t^{\alpha}$, we have

$$
E_{\alpha}\left(-\xi^{-k \alpha} t^{\alpha}\right)=-\xi^{-k \alpha} t^{\alpha} E_{\alpha, \alpha+1}\left(-\xi^{-k \alpha} t^{\alpha}\right)+1
$$

hence,

$$
-\xi^{-k \alpha} t^{\alpha} E_{\alpha, \alpha+1}\left(-\xi^{-k \alpha} t^{\alpha}\right)=E_{\alpha}\left(-\xi^{-k \alpha} t^{\alpha}\right)-1 .
$$


Inserting (49) into (47) yields

$$
y(t)=\lambda E_{\alpha}\left(-t^{\alpha}\right)+\lambda \sum_{i=1}^{\infty} \sum_{k=0}^{i} \frac{\xi^{i \alpha(1-i) / 2-i}}{G_{i}^{\prime}\left(-\xi^{-k \alpha}\right)}\left(E_{\alpha}\left(-\xi^{-k \alpha} t^{\alpha}\right)-1\right)
$$

which is the required exact solution. However, we can rewrite Eq. (50) as

$$
y(t)=\lambda E_{\alpha}\left(-t^{\alpha}\right)+\lambda\left(\sum_{i=1}^{\infty} \sum_{k=0}^{i} \frac{\xi^{i \alpha(1-i) / 2-i} E_{\alpha}\left(-\xi^{-k \alpha} t^{\alpha}\right)}{G_{i}^{\prime}\left(-\xi^{-k \alpha}\right)}-\sum_{i=1}^{\infty} \sum_{k=0}^{i} \frac{\xi^{i \alpha(1-i) / 2-i}}{G_{i}^{\prime}\left(-\xi^{-k \alpha}\right)}\right)
$$

or

$$
y(t)=\lambda E_{\alpha}\left(-t^{\alpha}\right)+\lambda\left(\sum_{i=1}^{\infty} \sum_{k=0}^{i} \frac{\xi^{i \alpha(1-i) / 2-i} E_{\alpha}\left(-\xi^{-k \alpha} t^{\alpha}\right)}{G_{i}^{\prime}\left(-\xi^{-k \alpha}\right)}-S\right),
$$

where $S$ is the sum defined by

$$
S=\sum_{i=1}^{\infty} \sum_{k=0}^{i} \frac{\xi^{i \alpha(1-i) / 2-i}}{G_{i}^{\prime}\left(-\xi^{-k \alpha}\right)}
$$

\section{The solution in a simplest form}

Here, we show that the sum $S$ in Eq. (53) vanishes, and hence the right-hand side of Eq. (52) can be further simplified. To do that, we express $S$ as

$$
S=\sum_{i=1}^{\infty} \xi^{i \alpha(1-i) / 2-i} \sum_{k=0}^{i} \frac{1}{G_{i}^{\prime}\left(-\xi^{-k \alpha}\right)}=\sum_{i=1}^{\infty} \xi^{i \alpha(1-i) / 2-i} \psi_{i}
$$

where

$$
\psi_{i}=\sum_{k=0}^{i} \frac{1}{G_{i}^{\prime}\left(-\xi^{-k \alpha}\right)}
$$

From (54), we have

$$
S=\xi^{-1} \psi_{1}+\xi^{-\alpha-2} \psi_{2}+\xi^{-3 \alpha-3} \psi_{3}+\cdots
$$

It is clear from (56) that $S$ vanishes when each $\psi_{i}$ vanishes, i.e., $\psi_{i}=0, \forall i \geq 1$. For $\psi_{1}$, we find

$$
\psi_{1}=\sum_{k=0}^{1} \frac{1}{G_{1}^{\prime}\left(-\xi^{-k \alpha}\right)}=\frac{1}{G_{1}^{\prime}(-1)}+\frac{1}{G_{1}^{\prime}\left(-\xi^{-\alpha}\right)} .
$$

From the definition of $G_{i}\left(s^{\alpha}\right)$, we have at $i=1$ that

$$
G_{1}\left(s^{\alpha}\right)=\prod_{k=0}^{1}\left(s^{\alpha}+\xi^{-k \alpha}\right)=\left(s^{\alpha}+1\right)\left(s^{\alpha}+\xi^{-\alpha}\right),
$$


i.e.,

$$
G_{1}\left(s^{\alpha}\right)=\left(s^{\alpha}\right)^{2}+\left(1+\xi^{-\alpha}\right) s^{\alpha}+\xi^{-\alpha}, G_{1}^{\prime}\left(s^{\alpha}\right)=2 s^{\alpha}+\left(1+\xi^{-\alpha}\right),
$$

and hence,

$$
G_{1}^{\prime}(-1)=-1+\xi^{-\alpha}, \quad G_{1}^{\prime}\left(-\xi^{-\alpha}\right)=1-\xi^{-\alpha} .
$$

Substituting (60) into (57), we obtain

$$
\psi_{1}=\frac{1}{-1+\xi^{-\alpha}}+\frac{1}{1-\xi^{-\alpha}}=0 .
$$

Similarly, we can prove that $\psi_{2}=0$, in this case we have

$$
\psi_{2}=\sum_{k=0}^{2} \frac{1}{G_{2}^{\prime}\left(-\xi^{-k \alpha}\right)}=\frac{1}{G_{2}^{\prime}(-1)}+\frac{1}{G_{2}^{\prime}\left(-\xi^{-\alpha}\right)}+\frac{1}{G_{2}^{\prime}\left(-\xi^{-2 \alpha}\right)}
$$

and

$$
G_{2}\left(s^{\alpha}\right)=\prod_{k=0}^{2}\left(s^{\alpha}+\xi^{-k \alpha}\right)=\left(s^{\alpha}+1\right)\left(s^{\alpha}+\xi^{-\alpha}\right)\left(s^{\alpha}+\xi^{-2 \alpha}\right),
$$

i.e.,

$$
\begin{aligned}
& G_{2}\left(s^{\alpha}\right)=\left(s^{\alpha}\right)^{3}+\left(1+\xi^{-\alpha}+\xi^{-2 \alpha}\right)\left(s^{\alpha}\right)^{2}+\left(\xi^{-\alpha}+\xi^{-2 \alpha}+\xi^{-3 \alpha}\right) s^{\alpha}+\xi^{-3 \alpha}, \\
& G_{2}^{\prime}\left(s^{\alpha}\right)=3\left(s^{\alpha}\right)^{2}+2\left(1+\xi^{-\alpha}+\xi^{-2 \alpha}\right) s^{\alpha}+\left(\xi^{-\alpha}+\xi^{-2 \alpha}+\xi^{-3 \alpha}\right) \\
& G_{2}^{\prime}(-1)=\left(1+\xi^{-\alpha}\right)\left(1-\xi^{-\alpha}\right)^{2} \\
& G_{2}^{\prime}\left(-\xi^{-\alpha}\right)=-\xi^{-\alpha}\left(1-\xi^{-\alpha}\right)^{2} \\
& G_{2}^{\prime}\left(-\xi^{-2 \alpha}\right)=\xi^{-\alpha}\left(1+\xi^{-\alpha}\right)\left(1-\xi^{-\alpha}\right)^{2} .
\end{aligned}
$$

Substituting (66)-(68) into (62), we obtain

$$
\begin{aligned}
\psi_{2} & =\frac{1}{\left(1+\xi^{-\alpha}\right)\left(1-\xi^{-\alpha}\right)^{2}}+\frac{1}{-\xi^{-\alpha}\left(1-\xi^{-\alpha}\right)^{2}}+\frac{1}{\xi^{-\alpha}\left(1+\xi^{-\alpha}\right)\left(1-\xi^{-\alpha}\right)^{2}} \\
& =\frac{\xi^{-\alpha}-\left(1+\xi^{-\alpha}\right)+1}{\xi^{-\alpha}\left(1+\xi^{-\alpha}\right)\left(1-\xi^{-\alpha}\right)^{2}} \\
& =0
\end{aligned}
$$

It can be proved by induction that $\psi_{i}=0, \forall i \geq 1$, and hence the sum $S$ in (56) vanishes. Formulas (59), (64), and (65) can also be obtained directly using the $q$-calculus [41], see the appendices. Therefore, solution (52) takes the form

$$
y(t)=\lambda E_{\alpha}\left(-t^{\alpha}\right)+\lambda \sum_{i=1}^{\infty} \sum_{k=0}^{i} \frac{\xi^{i \alpha(1-i) / 2-i} E_{\alpha}\left(-\xi^{-k \alpha} t^{\alpha}\right)}{G_{i}^{\prime}\left(-\xi^{-k \alpha}\right)} .
$$


Indeed, expression (70) can also be put in a simpler form by writing the initial component $\lambda E_{\alpha}\left(-t^{\alpha}\right)$ as

$$
\lambda E_{\alpha}\left(-t^{\alpha}\right)=\lambda\left[\sum_{k=0}^{i} \frac{\xi^{i \alpha(1-i) / 2-i} E_{\alpha}\left(-\xi^{-k \alpha} t^{\alpha}\right)}{G_{i}^{\prime}\left(-\xi^{-k \alpha}\right)}\right]_{i=0},
$$

where

$$
\begin{aligned}
& {\left[E_{\alpha}\left(-\xi^{-k \alpha} t^{\alpha}\right)\right]_{k=0}=E_{\alpha}\left(-t^{\alpha}\right), \quad\left[\xi^{i \alpha(1-i) / 2-i}\right]_{i=0}=1,} \\
& {\left[G_{i}^{\prime}\left(-\xi^{-k \alpha}\right)\right]_{k=0, i=0}=G_{0}^{\prime}(-1)=1 .}
\end{aligned}
$$

In view of (70) and (71), we obtain the solution in the simplest form:

$$
y(t)=\lambda \sum_{i=0}^{\infty} \sum_{k=0}^{i} \frac{\xi^{i \alpha(1-i) / 2-i} E_{\alpha}\left(-\xi^{-k \alpha} t^{\alpha}\right)}{G_{i}^{\prime}\left(-\xi^{-k \alpha}\right)} .
$$

\section{Validation as $\alpha \rightarrow 1$}

Here, it is shown that the exact solution obtained by Bakodah and Ebaid [4] for the SAE can be recovered as a special case of our exact solution (73) $\alpha \rightarrow 1$. In such a case, Eq. (73) reduces to

$$
y(t)=\lambda \sum_{i=0}^{\infty} \sum_{k=0}^{i} \frac{\xi^{i(1-i) / 2-i} E_{1}\left(-\xi^{-k} t\right)}{G_{i}^{\prime}\left(-\xi^{-k}\right)}
$$

i.e.,

$$
y(t)=\lambda \sum_{i=0}^{\infty} \sum_{k=0}^{i} \frac{\xi^{-i(1+i) / 2} e^{-\xi^{-k} t}}{G_{i}^{\prime}\left(-\xi^{-k}\right)}, \quad\left[G_{i}\left(s^{\alpha}\right)\right]_{\alpha \rightarrow 1}=G_{i}(s)=\prod_{k=0}^{i}\left(s+\xi^{-k}\right) .
$$

However, $G_{i}(s)$ can be written as

$$
G_{i}(s)=\prod_{k=0}^{i}\left(s+\xi^{-k}\right)=\prod_{k=0}^{i} \xi^{-k} \prod_{k=0}^{i}\left(\xi^{k} s+1\right)=\xi^{-i(1+i) / 2} Q_{i}(s),
$$

where $Q_{i}(s)$ is defined as

$$
Q_{i}(s)=\prod_{k=0}^{i}\left(\xi^{k} s+1\right)
$$

From (76), we obtain

$$
G_{i}^{\prime}(s)=\xi^{-i(1+i) / 2} Q_{i}^{\prime}(s)
$$

which leads to

$$
G_{i}^{\prime}\left(-\xi^{-k}\right)=\xi^{-i(1+i) / 2} Q_{i}^{\prime}\left(-\xi^{-k}\right)
$$


Substituting (79) into (75), we obtain

$$
y(t)=\lambda \sum_{i=0}^{\infty} \sum_{k=0}^{i} \frac{e^{-\xi^{-k} t}}{Q_{i}^{\prime}\left(-\xi^{-k}\right)}
$$

which is the corresponding solution obtained by Bakodah and Ebaid [4] for the SAE. Here, it may be important to refer to that the present exact solution (73) for the FAE is introduced for the first time. Moreover, the current analysis was not previously reported on the FAE.

\section{Convergence analysis}

Theorem 3 For $\alpha \in(0,1]$, the closed-form series solution (73) is convergent $\forall \xi>1, t \geq 0$.

Proof Firstly, we rewrite (73) as

$$
y(t)=\sum_{i=0}^{\infty} c_{i}
$$

where $c_{i}$ is defined by

$$
c_{i}=\lambda \sum_{k=0}^{i} \frac{\xi^{i \alpha(1-i) / 2-i} E_{\alpha}\left(-\xi^{-k \alpha} t^{\alpha}\right)}{G_{i}^{\prime}\left(-\xi^{-k \alpha}\right)} .
$$

The series coefficient $c_{i}$ can be rewritten as

$$
c_{i}=\lambda \sum_{k=0}^{i} \frac{q^{-i(1-i) / 2+i / \alpha} E_{\alpha}\left(-q^{k} \tau\right)}{G_{i}^{\prime}\left(-q^{k}\right)}, \quad q=\xi^{-\alpha}, \tau=t^{\alpha} .
$$

Accordingly,

$$
c_{i+1}=\lambda \sum_{k=0}^{i+1} \frac{q^{(i+1)(i / 2+1 / \alpha)} E_{\alpha}\left(q^{k} \tau\right)}{G_{i+1}^{\prime}\left(-q^{k}\right)}
$$

or

$$
c_{i+1}=\lambda \sum_{k=0}^{i} \frac{q^{(i+1)(i / 2+1 / \alpha)} E_{\alpha}\left(-q^{k} \tau\right)}{G_{i+1}^{\prime}\left(-q^{k}\right)}+\lambda \frac{q^{(i+1)(i / 2+1 / \alpha)} E_{\alpha}\left(-q^{i+1} \tau\right)}{G_{i+1}^{\prime}\left(-q^{i+1}\right)} .
$$

From the definition of $G_{i}\left(s^{\alpha}\right)$ in (26), we find that

$$
G_{i}\left(s^{\alpha}\right)=\prod_{k=0}^{i}\left(s^{\alpha}+q^{k}\right), \quad G_{i+1}\left(s^{\alpha}\right)=\prod_{k=0}^{i+1}\left(s^{\alpha}+q^{k}\right)=\left(s^{\alpha}+q^{i+1}\right) G_{i}\left(s^{\alpha}\right),
$$

and

$$
G_{i+1}^{\prime}\left(s^{\alpha}\right)=\left(s^{\alpha}+q^{i+1}\right) G_{i}^{\prime}\left(s^{\alpha}\right)+G_{i}\left(s^{\alpha}\right) .
$$


Hence,

$$
G_{i+1}^{\prime}\left(-q^{k}\right)=\left(-q^{k}+q^{i+1}\right) G_{i}^{\prime}\left(-q^{k}\right)+G_{i}\left(-q^{k}\right)=\left(q^{i+1}-q^{k}\right) G_{i}^{\prime}\left(-q^{k}\right)
$$

where $G_{i}\left(-q^{k}\right)=0(\forall k=0,1, \ldots, i)$. Moreover, we have from (87) that $G_{i+1}^{\prime}\left(-q^{i+1}\right)=$ $G_{i}\left(-q^{i+1}\right)$ and, consequently,

$$
c_{i+1}=\lambda \sum_{k=0}^{i} \frac{q^{(i+1)(i / 2+1 / \alpha)} E_{\alpha}\left(-q^{k} \tau\right)}{\left(q^{i+1}-q^{k}\right) G_{i}^{\prime}\left(-q^{k}\right)}+\lambda \frac{q^{(i+1)(i / 2+1 / \alpha)} E_{\alpha}\left(-q^{i+1} \tau\right)}{G_{i}\left(-q^{i+1}\right)} .
$$

From (83) and (89), we have

$$
c_{i+1}-c_{i}=\lambda \sum_{k=0}^{i}\left(\frac{\mu^{(i+1)(i / 2+1 / \alpha)}}{q^{i+1}-q^{k}}-q^{-i(1-i) / 2+i / \alpha}\right) \frac{E_{\alpha}\left(-q^{k} \tau\right)}{G_{i}^{\prime}\left(-q^{k}\right)}+\lambda \frac{q^{(i+1)(i / 2+1 / \alpha)} E_{\alpha}\left(-q^{i+1} \tau\right)}{G_{i}\left(-q^{i+1}\right)}
$$

which can be simplified as

$$
c_{i+1}-c_{i}=\lambda q^{i(i-1) / 2+i / \alpha} \sum_{k=0}^{i} \frac{\Omega_{i, k} E_{\alpha}\left(-q^{k} \tau\right)}{G_{i}^{\prime}\left(-q^{k}\right)}+\lambda q^{(i+1) / \alpha} \frac{E_{\alpha}\left(-q^{i+1} \tau\right)}{\prod_{k=0}^{i}\left(1-q^{i-k+1}\right)},
$$

where

$$
\Omega_{i, k}=-\frac{q^{k}-\left(1-q^{1 / \alpha}\right) q^{i}}{q^{k}-q^{i+1}}, \quad G_{i}\left(-q^{i+1}\right)=q^{i(i+1) / 2} \prod_{k=0}^{i}\left(1-q^{i-k+1}\right) .
$$

Using $q$-calculus notations, we have $\prod_{k=0}^{i}\left(1-q^{i-k+1}\right)=\prod_{j=0}^{i}\left(1-q \cdot q^{j}\right)=(q: q)_{i+1}$ (see Appendix A). For a fixed integer $i \geq 1$, we have from (91) that

$$
\left|c_{i+1}-c_{i}\right| \leq \lambda q^{i(i-1) / 2+i / \alpha} \sum_{k=0}^{i}\left|\frac{\Omega_{i, k}}{G_{i}^{\prime}\left(-q^{k}\right)}\right|\left|E_{\alpha}\left(-q^{k} \tau\right)\right|+\frac{\lambda q^{(i+1) / \alpha}}{\left|(q: q)_{i+1}\right|}\left|E_{\alpha}\left(-q^{i+1} \tau\right)\right| .
$$

Applying the property $0<E_{\alpha}(-\gamma \tau) \leq 1(\gamma>0, \tau \geq 0)$ on (93), it then follows

$$
\left|c_{i+1}-c_{i}\right| \leq \lambda q^{i(i-1) / 2+i / \alpha} \sum_{k=0}^{i}\left|\frac{\Omega_{i, k}}{G_{i}^{\prime}\left(-q^{k}\right)}\right|+\frac{\lambda q^{(i+1) / \alpha}}{\left|(q: q)_{i+1}\right|} .
$$

As $i \rightarrow \infty$ and since $q<1$, then $q^{i(i-1) / 2+i / \alpha} \rightarrow 0, q^{(i+1) / \alpha} \rightarrow 0(\forall \alpha \in(0,1])$ and $0<(q: q)_{\infty}<$ 1 , hence $\left|c_{i+1}-c_{i}\right| \rightarrow 0$ which completes the proof.

\section{Numerical results and discussions}

This section is devoted to performing several comparisons with the existing solution in the relevant literature using the CAS Wolfram Mathematica. For numerical purposes, we define the $n$-term approximate solution $\sigma_{n}$ of series (73) as follows:

$$
\sigma_{n}(t)=\lambda \sum_{i=0}^{n-1} \sum_{k=0}^{i} \frac{\xi^{i \alpha(1-i) / 2-i} E_{\alpha}\left(-\xi^{-k \alpha} t^{\alpha}\right)}{G_{i}^{\prime}\left(-\xi^{-k \alpha}\right)}, \quad n \geq 1 .
$$




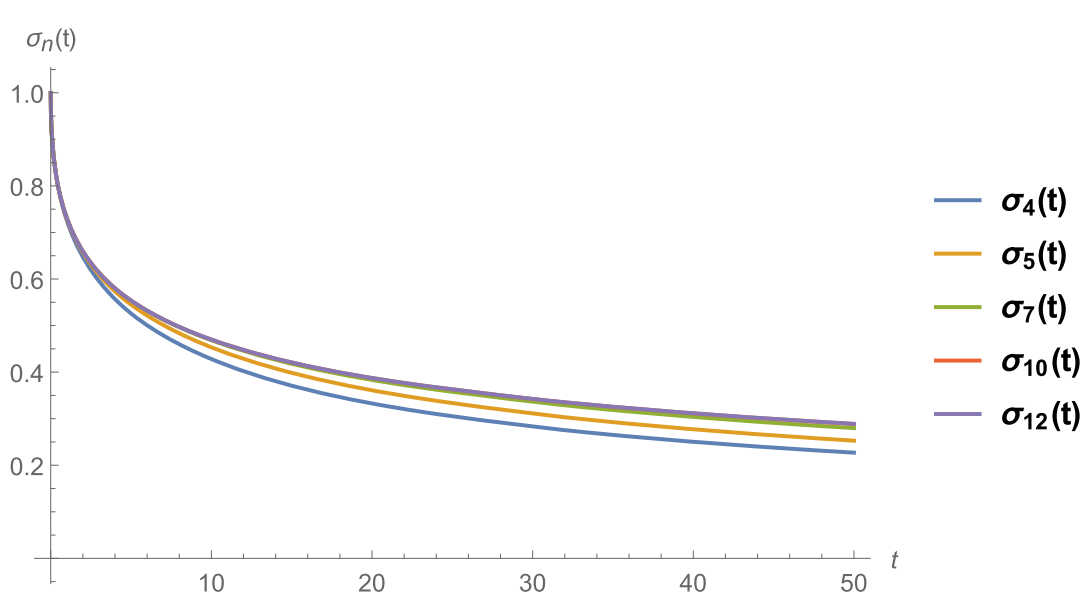

Figure 1 Convergence of current approximate solutions $\sigma_{n}(t), n=4,5,7,10,12$, at $\lambda=1, \xi=1.5$, and $\alpha=0.5$

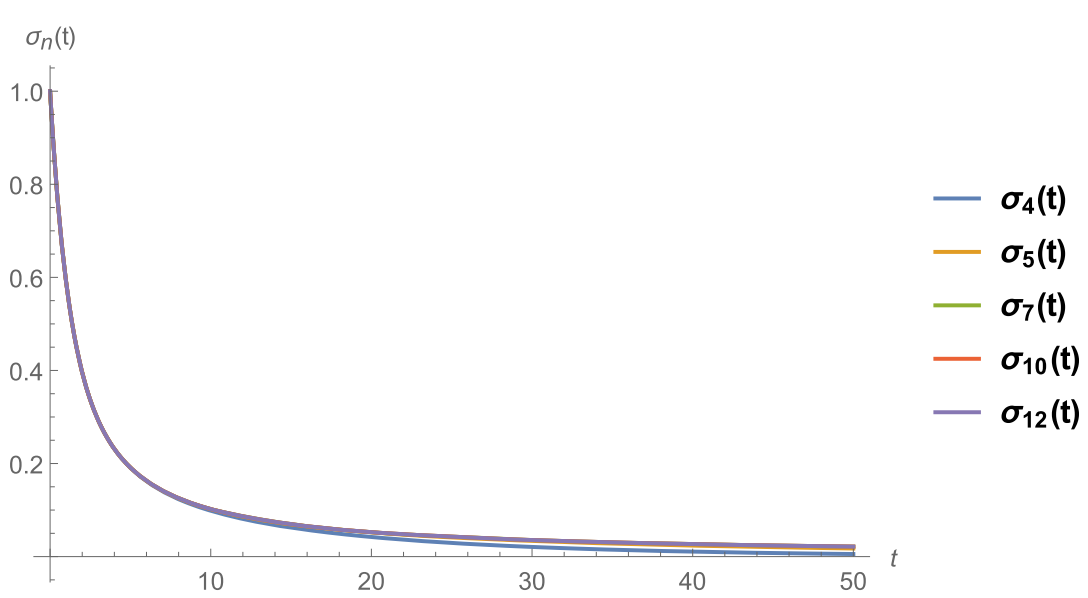

Figure 2 Convergence of current approximate solutions $\sigma_{n}(t), n=4,5,7,10,12$, at $\lambda=1, \xi=2.5$, and $\alpha=1$

Figures 1 and 2 display the convergence of current approximate solutions $\sigma_{n}(t), n=$ $4,5,7,10,12$, at $\lambda=1, \xi=2.5, \alpha=0.5$ (Fig. 1), and $\alpha=1$ (Fig. 2).

In the literature, Bhalekar and Patade [42] solved the initial value problem (IVP)

$$
{ }_{0}^{C} D_{t}^{\alpha} y(t)=A y(t)+B y(C t), \quad y(1)=1,0<\alpha \leq 1, A \in \mathbb{R}, B \in \mathbb{R}
$$

where $C$ is a constant and $C \in(0,1)$. In [41], the solution of IVP (96) was given as

$$
y(t)=1+\sum_{i=1}^{\infty} \frac{t^{i \alpha}}{\Gamma(i \alpha+1)} \prod_{k=0}^{i-1}\left(A+B C^{\alpha k}\right) .
$$

Comparing (96) with Eqs. (1)-(2), we find that $\lambda=1, A=-1$, and $B=C=\frac{1}{\xi}$. Accordingly, Eq. (97) becomes

$$
y(t)=1+\sum_{i=1}^{\infty} \frac{t^{i \alpha}}{\Gamma(i \alpha+1)} \prod_{k=0}^{i-1}\left(\xi^{-\alpha k-1}-1\right)
$$




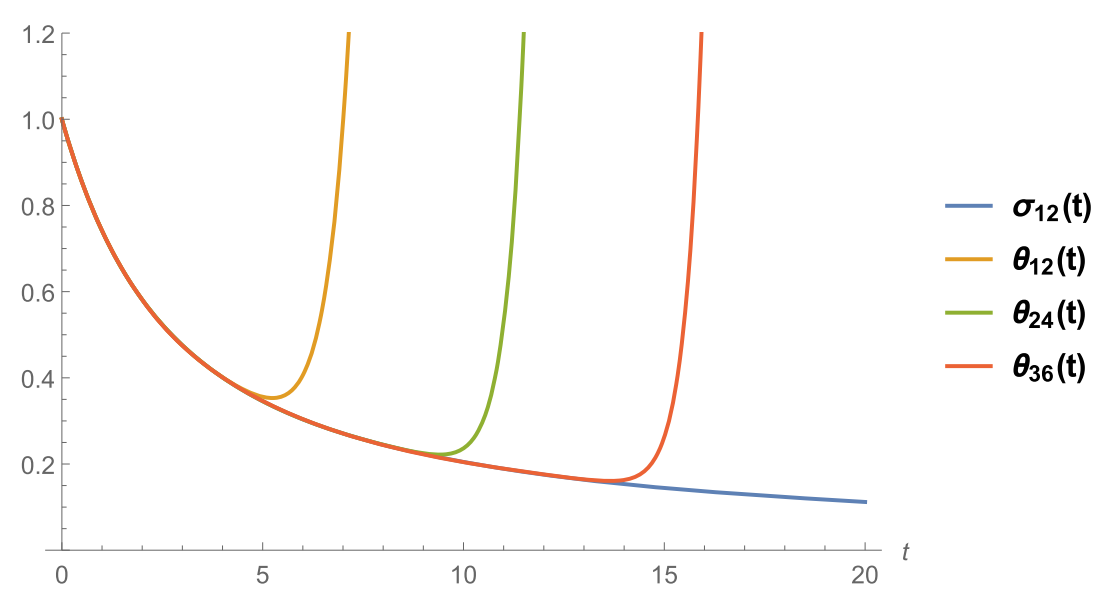

Figure 3 Comparison between $\sigma_{12}$ (present) and $\theta_{12}, \theta_{24}$, and $\theta_{36}$ in Ref. [42] at $\lambda=1, \xi=1.5$, and $\alpha=0.5$

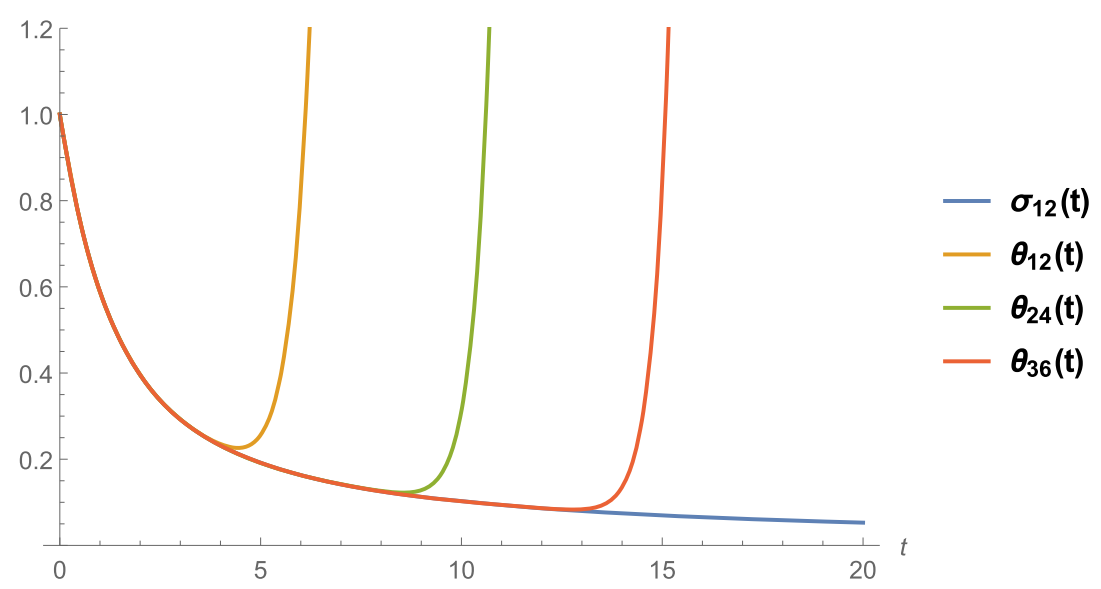

Figure 4 Comparison between $\sigma_{12}$ (present) and $\theta_{12}, \theta_{24}$ in Ref. [42], and $\theta_{36}$ at $\lambda=1, \xi=2.5$, and $\alpha=1$

with the $n$-term approximate solution $\theta_{n}(t)$ defined by

$$
\theta_{n}(t)=1+\sum_{i=1}^{n} \frac{t^{i \alpha}}{\Gamma(i \alpha+1)} \prod_{k=0}^{i-1}\left(\xi^{-\alpha k-1}-1\right), \quad n \geq 1
$$

Now the task is to compare the present approximation (95) and the corresponding one in Ref. [42] given by (99). For fixed $\lambda=1$ and $\xi=1.5$, the comparisons between the present $\sigma_{12}$ and $\theta_{12}, \theta_{24}, \theta_{36}$ of Ref. [42] are depicted in Figs. 3, 4 at $\alpha=0.5$ and $\alpha=1$, respectively. Figures 3 and 4 show that the approximation $\theta_{12}$ coincides with the present one $\sigma_{12}$ on the interval [0,5), while $\theta_{24}$ coincides with our $\sigma_{12}$ on a slightly wider interval $[0,10)$, and $\theta_{36}$ leads to a coincidence on the interval $[0,15)$. It is observed that the number of terms needed from $\theta_{n}$ to achieve a coincidence with our exact solution is multiplied by the present number of terms of $\sigma_{n}$. Therefore, the obtained results confirm the effectiveness and efficiency of the present approach. 


\section{Conclusions}

The Heaviside expansion formula in CC was extended in this paper in view of FC. Several theoretical theorems and lemmas were proved for the extended Heaviside expansion formula and then applied on particular expressions in FC. Accordingly, the solution of the FAE was obtained based on Caputo's fractional derivative. The solution was derived in a simplest form in terms of a one-parameter Mittag-Leffler function. Besides, the convergence of the obtained solution was theoretically proved. Furthermore, it was shown that the exact solution obtained by Bakodah and Ebaid [4] for the SAE was recovered as a special case of the present exact one for the FAE when the fractional order tends to one.

In addition, graphical comparisons with another approach in the literature were performed. The advantage of the present analysis over the existing one in the relevant literature was discussed and analyzed. It was also shown that the current solution converges in the whole domain, as consequences of the properties of the Mittag-Leffler functions, while the solution in Ref. [42] converges in subdomains.

\section{Appendix A: Concepts of $q$-calculus}

For $q \in(0,1)$ and $x \in \mathbb{N}$, the $q$-version of $x$ is defined as (see [41])

$$
[x]_{q}=\frac{1-q^{x}}{1-q} \text { and } \quad \lim _{q \rightarrow 1}[x]_{q}=x
$$

For $i, k \in \mathbb{N}$, the $q$-binomial $\left(\begin{array}{l}i \\ k\end{array}\right)_{q}$ is defined by

$$
\left(\begin{array}{l}
i \\
k
\end{array}\right)_{q}=\frac{[i]_{q} !}{[k]_{q} ![i-k]_{q} !} \text { and }\left(\begin{array}{l}
i \\
0
\end{array}\right)_{q}=\left(\begin{array}{l}
i \\
i
\end{array}\right)_{q}=1
$$

where $[i]_{q}$ ! is the $q$-factorial of $i$ :

$$
[i]_{q} !=[1]_{q} \cdot[2]_{q} \cdot[3]_{q} \ldots[i]_{q} \text { and } \lim _{q \rightarrow 1}[i]_{q} !=[i] !
$$

The $q$-shifted factorials are defined by

$$
\begin{aligned}
& (a: q)_{i}=\prod_{k=0}^{i-1}\left(1-a q^{k}\right), \quad \text { where }(a: q)_{0}=1, \quad \text { and } \\
& (a: q)_{\infty}=\lim _{i \rightarrow \infty}(a: q)_{i}=\prod_{k=0}^{\infty}\left(1-a q^{k}\right) .
\end{aligned}
$$

For $u, v \in \mathbb{R}$ and $i \in \mathbb{N}$, the $q$-binomial theorem is given by

$$
(u+v)_{q}^{i}=\prod_{k=0}^{i-1}\left(u+q^{k} v\right)=\sum_{r=0}^{i} q^{\left(\begin{array}{c}
r \\
2
\end{array}\right)}\left(\begin{array}{l}
i \\
r
\end{array}\right)_{q} u^{i-r} v^{r} .
$$




\section{Appendix B: Properties of $G_{i}\left(s^{\alpha}\right)$ in view of $q$-calculus}

Here, we show that the $q$-calculus can be implemented to derive some obtained results in Sect. 5. Firstly, we rewrite $G_{i}\left(s^{\alpha}\right)$ in Eq. (26) in view of $q$-calculus as follows:

$$
G_{i}\left(s^{\alpha}\right)=\prod_{k=0}^{i}\left(s^{\alpha}+q^{k}\right), \quad 0<q=\xi^{-\alpha}<1 .
$$

Substituting $u=s^{\alpha}$ and $v=1$ in (A.5) and replacing $i$ with $i+1$, we obtain

$$
\left(s^{\alpha}+1\right)_{q}^{i+1}=\prod_{k=0}^{i}\left(s^{\alpha}+q^{k}\right)=\sum_{r=0}^{i+1} q^{\left(\begin{array}{c}
r \\
2
\end{array}\right)}\left(\begin{array}{c}
i+1 \\
r
\end{array}\right)_{q}\left(s^{\alpha}\right)^{i-r+1} .
$$

In view of (B.1) and (B.2) we have the following series form for $G_{i}\left(s^{\alpha}\right)$ :

$$
G_{i}\left(s^{\alpha}\right)=\sum_{r=0}^{i+1} q^{\left(\begin{array}{c}
r \\
2
\end{array}\right)}\left(\begin{array}{c}
i+1 \\
r
\end{array}\right)_{q}\left(s^{\alpha}\right)^{i-r+1}
$$

Differentiating (B.3) once with respect to $s^{\alpha}$, we get

$$
G_{i}^{\prime}\left(s^{\alpha}\right)=\sum_{r=0}^{i+1} q^{\left(\begin{array}{c}
r \\
2
\end{array}\right)}\left(\begin{array}{c}
i+1 \\
r
\end{array}\right)_{q}(i-r+1)\left(s^{\alpha}\right)^{i-r}=\sum_{r=0}^{i} q^{\frac{r}{2}(r-1)}\left(\begin{array}{c}
i+1 \\
r
\end{array}\right)_{q}(i-r+1)\left(s^{\alpha}\right)^{i-r} .
$$

This is a unified formula to calculate $G_{i}^{\prime}\left(s^{\alpha}\right)$ for fixed $i$. For example, at $i=1$, we obtain

$$
G_{1}^{\prime}\left(s^{\alpha}\right)=\sum_{r=0}^{1} q^{\frac{r}{2}(r-1)}\left(\begin{array}{l}
2 \\
r
\end{array}\right)_{q}(2-r)\left(s^{\alpha}\right)^{1-r}=2\left(\begin{array}{l}
2 \\
0
\end{array}\right)_{q}\left(s^{\alpha}\right)+\left(\begin{array}{l}
2 \\
1
\end{array}\right)_{q}
$$

and hence,

$$
G_{1}^{\prime}\left(s^{\alpha}\right)=2\left(s^{\alpha}\right)+(1+q)=2\left(s^{\alpha}\right)+1+\xi^{-\alpha},
$$

which is the same expression in Eq. (59), where

$$
\left(\begin{array}{l}
2 \\
0
\end{array}\right)_{q}=1, \quad\left(\begin{array}{l}
2 \\
1
\end{array}\right)_{q}=1+q=1+\xi^{-\alpha} .
$$

Similarly, at $i=2$ we have

$$
G_{2}^{\prime}\left(s^{\alpha}\right)=\sum_{r=0}^{2} q^{\frac{r}{2}(r-1)}\left(\begin{array}{l}
3 \\
r
\end{array}\right)_{q}(3-r)\left(s^{\alpha}\right)^{2-r}=3\left(\begin{array}{l}
3 \\
0
\end{array}\right)_{q}\left(s^{\alpha}\right)^{2}+2\left(\begin{array}{l}
3 \\
1
\end{array}\right)_{q}\left(s^{\alpha}\right)+q\left(\begin{array}{l}
3 \\
2
\end{array}\right)_{q},
$$

i.e.,

$$
\begin{aligned}
G_{2}^{\prime}\left(s^{\alpha}\right) & =3\left(s^{\alpha}\right)^{2}+2\left(1+q+q^{2}\right)\left(s^{\alpha}\right)+q\left(1+q+q^{2}\right), \\
& =3\left(s^{\alpha}\right)^{2}+2\left(1+\xi^{-\alpha}+\xi^{-2 \alpha}\right)\left(s^{\alpha}\right)+\left(\xi^{-\alpha}+\xi^{-2 \alpha}+\xi^{-3 \alpha}\right),
\end{aligned}
$$


where

$$
\left(\begin{array}{l}
3 \\
0
\end{array}\right)_{q}=1, \quad\left(\begin{array}{l}
3 \\
1
\end{array}\right)_{q}=\left(\begin{array}{l}
3 \\
2
\end{array}\right)_{q}=1+q+q^{2}=1+\xi^{-\alpha}+\xi^{-2 \alpha}
$$

The expression given by Eq. (B.9) agrees with the previous one in Eq. (65). Indeed, the unified formula (B.4) for $G_{i}^{\prime}\left(s^{\alpha}\right)$ is easily programmable by any software when compared with the preceding one in Sect. 5. This of course reflects the advantages of the $q$-calculus.

Acknowledgements

The authors would like to thank the referees for their valuable comments and suggestions which helped to improve the manuscript.

Funding

Not applicable.

Availability of data and materials

Not applicable.

Competing interests

The authors declare that they have no competing interests.

\section{Authors' contributions}

This work was carried out in collaboration among all authors. All authors read and approved the final manuscript.

\section{Author details}

${ }^{1}$ Department of Mathematics, Faculty of Science, University of Tabuk, P.O. Box 741, Tabuk, 71491, Saudi Arabia.

${ }^{2}$ Engineering School (DEIM), University of Tuscia, Viterbo, Italy. ${ }^{3}$ Department of Mathematics, Faculty of Sciences and Humanities, Prince Sattam Bin Abdulaziz University, Alkharj, 11942, Saudi Arabia. ${ }^{4}$ Department of Basic Engineering

Science, Faculty of Engineering, Menofia University, Shebin El-Kom, 32511, Egypt.

\section{Publisher's Note}

Springer Nature remains neutral with regard to jurisdictional claims in published maps and institutional affiliations.

Received: 12 October 2020 Accepted: 12 January 2021 Published online: 29 January 2021

\section{References}

1. Ambartsumian, V.A.: On the fluctuation of the brightness of the milky way. Dokl. Akad. Nauk SSSR 44, $223-226$ (1994)

2. Kato, T., McLeod, J.B.: The functional-differential equation $y^{\prime}(x)=a y(\lambda x)+b y(x)$. Bull. Am. Math. Soc. 77, 891-935 (1971)

3. Patade, J., Bhalekar, S.: On analytical solution of Ambartsumian equation. Nat. Acad. Sci. Lett. 40, 291-293 (2017)

4. Bakodah, H.O., Ebaid, A.: Exact solution of Ambartsumian delay differential equation and comparison with Daftardar-Gejji and Jafari approximate method. Mathematics 6, 331 (2018)

5. Alatawi, A.A., Aljoufi, M., Alharbi, F.M., Ebaid, A.: Investigation of the surface brightness model in the milky way via homotopy perturbation method. J. Appl. Math. Phys. 8(3), 434-442 (2020)

6. Khaled, S.M., El-Zahar, E.R., Ebaid, A.: Solution of Ambartsumian delay differential equation with conformable derivative. Mathematics 7, 425 (2019)

7. Kumar, D., Singh, J., Baleanu, D., et al.: Analysis of a fractional model of the Ambartsumian equation. Eur. Phys. J. Plus 133, 133-259 (2018)

8. Adomian, G., Rach, R.: On the solution of algebraic equations by the decomposition method. J. Math. Anal. Appl. 105, 141-166 (1985)

9. Adomian, G., Rach, R.: Algebraic equations with exponential terms. J. Math. Anal. Appl. 112(1), 136-140 (1985)

10. Adomian, G., Rach, R.: Algebraic computation and the decomposition method. Kybernetes 15(1), 33-37 (1986)

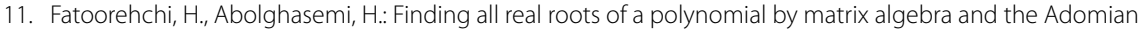
decomposition method. J. Egypt. Math. Soc. 22, 524-528 (2014)

12. Alshaery, A., Ebaid, A.: Accurate analytical periodic solution of the elliptical Kepler equation using the Adomian decomposition method. Acta Astronaut. 140, 27-33 (2017)

13. Adomian, G.: Solving Frontier Problems of Physics: The Decomposition Method. Kluwer Acad, Boston (1994)

14. Wazwaz, A.M.: Adomian decomposition method for a reliable treatment of the Bratu-type equations. Appl. Math. Comput. 166, 652-663 (2005)

15. Wazwaz, A.M.: The combined Laplace transform-Adomian decomposition method for handling nonlinear Volterra integro-differential equations. Appl. Math. Comput. 216, 1304-1309 (2010)

16. Ebaid, A.: Approximate analytical solution of a nonlinear boundary value problem and its application in fluid mechanics. Z. Naturforsch. A 66, 423-426 (2011)

17. Duan, J.S., Rach, R.: A new modification of the Adomian decomposition method for solving boundary value problems for higher order nonlinear differential equations. Appl. Math. Comput. 218, 4090-4118 (2011) 
18. Ebaid, A.: A new analytical and numerical treatment for singular two-point boundary value problems via the Adomian decomposition method. J. Comput. Appl. Math. 235, 1914-1924 (2011)

19. Wazwaz, A.M., Rach, R., Duan, J.S.: Adomian decomposition method for solving the Volterra integral form of the Lane-Emden equations with initial values and boundary conditions. Appl. Math. Comput. 219, 5004-5019 (2013)

20. Ali, E.H., Ebaid, A., Rach, R.: Advances in the Adomian decomposition method for solving two-point nonlinear boundary value problems with Neumann boundary conditions. Comput. Math. Appl. 63, 1056-1065 (2012)

21. Sheikholeslami, M., Ganji, D.D., Ashorynejad, H.R.: Investigation of squeezing unsteady nanofluid flow using ADM. Powder Technol. 239, 259-265 (2013)

22. Chun, C., Ebaid, A., Lee, M., Aly, E.H.: An approach for solving singular two point boundary value problems: analytical and numerical treatment. ANZIAM J. 53, 21-43 (2012)

23. Kashkari, B.S., Bakodah, H.O.: New modification of Laplace decomposition method for seventh order KdV equation. Appl. Math. Inf. Sci. 9(5), 2507-2512 (2015)

24. Ebaid, A., Aljoufi, M.D., Wazwaz, A.M.: An advanced study on the solution of nanofluid flow problems via Adomian's method. Appl. Math. Lett. 46, 117-122 (2015)

25. Bhalekar, S., Patade, J.: An analytical solution of fishers equation using decomposition method. Am. J. Comput. Appl. Math. 6, 123-127 (2016)

26. Bakodah, H.O., Al-Zaid, N.A., Mirzazadeh, M., Zhou, Q.: Decomposition method for solving Burgers' equation with Dirichlet and Neumann boundary conditions. Optik 130, 1339-1346 (2017)

27. Ebaid, A., Al-Enazi, A., Albalawi, B.Z., Aljoufi, M.D.: Accurate approximate solution of Ambartsumian delay differential equation via decomposition method. Math. Comput. Appl. 24(1), 7 (2019)

28. Kaur, D., Agarwal, P., Rakshit, M., Chand, M.: Fractional calculus involving (p,q)-Mathieu type series. Appl. Math. Nonlinear Sci. 5(2), 15-34 (2020)

29. Agarwal, P., Mondal, S.R., Nisar, K.S.: On fractional integration of generalized Struve functions of first kind. Thai J. Math. (2021, to appear)

30. Agarwal, P., Singh, R.: Modelling of transmission dynamics of Nipah virus (Niv): a fractional order approach. Phys. A, Stat. Mech. Appl. 547(1), 124243 (2020)

31. Alderremy, A.A., Saad, K.M., Agarwal, P., Aly, S., Jain, S.: Certain new models of the multi space-fractional Gardner equation. Phys. A, Stat. Mech. Appl. 545(1), 123806 (2020)

32. Feng, Y.-Y., Yang, X.-J., Liu, J.-G., Chen, Z.-Q.: New perspective aimed at local fractional order memristor model on Cantor sets, Fractals (2021, to appear). https://doi.org/10.1142/S0218348X21500110

33. Feng, Y.-Y., Yang, X.-J., Liu, J.-G.: On overall behavior of Maxwell mechanical model by the combined Caputo fractional derivative. Chin. J. Phys. 66, 269-276 (2020)

34. Sweilam, N.H., Al-Mekhlafi, S.M., Assiri, T., Atangana, A.: Optimal control for cancer treatment mathematical model using Atangana-Baleanu-Caputo fractional derivative. Adv. Differ. Equ. 2020, 334 (2020). https://doi.org/10.1186/s13662-020-02793-9

35. Atangana, A., Qureshi, S.: Mathematical modeling of an autonomous nonlinear dynamical system for malaria transmission using Caputo derivative. In: Fractional Order Analysis: Theory, Methods and Applications (2020). https://doi.org/10.1002/9781119654223.ch9

36. Agarwal, P., El-Sayed, A.A.: Vieta-Lucas polynomials for solving a fractional-order mathematical physics model. Adv. Differ. Equ. 2020, 626 (2020). https://doi.org/10.1186/s13662-020-03085-y

37. Yassen, M.F., Attiya, A.A., Agarwal, P.: Subordination and superordination properties for certain family of analytic functions associated with Mittag-Leffler function. Symmetry 12, 1724 (2020)

38. Agarwal, P., El-Sayed, A.A., Tariboon, J.: Vieta-Fibonacci operational matrices for spectral solutions of variable-order fractional integro-differential equations. J. Comput. Appl. Math. 382, 113063 (2021)

39. Podlubny, I.: Fractional Differential Equations. Academic Press, San Diego (1999)

40. Spiegel, M.R.: Laplace Transforms. McGraw-Hill, New York (1965)

41. Chung, W.S., Kim, T., Kwon, H.: On the q-analog of the Laplace transform (English summary). Russ. J. Math. Phys. 21(2), 156-168 (2014)

42. Bhalekar, S., Patade, J.: Series solution of the pantograph equation and its properties. Fractal Fract. 1, $16(2017)$

\section{Submit your manuscript to a SpringerOpen ${ }^{\circ}$ journal and benefit from:}

- Convenient online submission

- Rigorous peer review

- Open access: articles freely available online

- High visibility within the field

- Retaining the copyright to your article

Submit your next manuscript at $\boldsymbol{s p r i n g e r o p e n . c o m ~}$ 